\title{
ESPIRONOLACTONA OU CLONIDINA COMO QUARTO FÁRMACO NA HIPERTENSÃO RESISTENTE?
}

\author{
SPIRONOLACTONE OR CLONIDINE AS THE FOURTH DRUG TO TREAT \\ RESISTANT HYPERTENSION?
}

Daniel S.S. Mello', Julia S. Gomes', Audes D. M. Feitosa²,3, José R. Matos-Souza', Wilson Nadruz'

\section{RESUMO}

A hipertensão arterial resistente (HAR) é definida como a ausência de controle pressórico nas medidas de pressão arterial (PA) de consultório a despeito do uso de três ou mais anti-hipertensivos em doses adequadas, incluindo-se preferencialmente um diurético, ou o controle pressórico atingido às custas do uso de quatro ou mais medicamentos. O uso de espironolactona, um antagonista dos receptores de aldosterona, como quarto fármaco no tratamento da HAR é indicado pelas principais diretrizes sobre o assunto, e tem a sua eficácia comprovada em ensaios clínicos e meta análises. Um estudo comparou o uso de clonidina, um agonista adrenérgico alfa-2, como quarto fármaco para tratamento da HAR em comparação com a espironolactona. Embora o desfecho primário (taxa de controle da PA no consultório ou na medida ambulatorial da PA) tenha sido similar com as duas medicações, a espironolactona mostrou maior redução na PA de 24 h quando comparada à clonidina. Neste contexto, a clonidina pode ser uma alternativa à espironolactona, particularmente em grupos específicos de pacientes que tenham contraindicação ao uso de espironolactona, como os que apresentam hipercalemia ou doença renal crônica pré dialítica.

Descritores: Hipertensão Resistente; Espironolactona; Clonidina.

\section{ABSTRACT}

Resistant Hypertension (RH) is defined as the absence of blood pressure (BP) control despite the use of three antihypertensive drugs in adequate doses, or the achievement of BP control with the use of four or more medications. The use of spironolactone, an antagonist of aldosterone receptors, as the fourth medication in the treatment of RH is recommended by current Management of Hypertension Guidelines, and its efficacy has been proved in clinical trials and meta-analysis. One clinical trial compared the use of clonidine, an adrenergic alpha-2 agonist, versus spironolactone as an option as the fourth drug in the treatment of RH. The results showed similar rates of the primary outcome (BP control at the office and at ambulatory monitoring) with both drugs, although spironolactone promoted greater reduction in $24 \mathrm{~h} \mathrm{BP}$ when compared with clonidine. In this context, clonidine can be used as an alternative to spironolactone, particularly among specific groups of patients that have contraindications to the use of spironolactone, such as patients with hyperkalemia or end stage renal disease.

Keywords: Resistant Hypertension; Spironolactone; Clonidine.

\section{HIPERTENSÃO ARTERIAL RESISTENTE}

A hipertensão arterial resistente (HAR) é definida como a ausência de controle pressórico nas medidas de pressão arterial (PA) de consultório a despeito do uso de três ou mais anti-hipertensivos em doses adequadas, incluindo-se preferencialmente um diurético, ou o controle pressórico atingido às custas do uso de quatro ou mais medicamentos. ${ }^{1-3}$

Estudos populacionais estimam a prevalência de HAR em
$12 \%$ da população hipertensa. ${ }^{2} \mathrm{O}$ diagnóstico é feito após a confirmação da adesão medicamentosa correta e a inclusão de um diurético apropriado. ${ }^{1-3}$ A 7ạ Diretriz Brasileira de Hipertensão Arterial recomenda a medida ambulatorial da PA (MAPA) ou a medida residencial da PA (MRPA) e a verificação sistemática de adesão para a realização do diagnóstico. ${ }^{4}$

Os fatores causais relacionados à presença de HAR incluem maior sensibilidade ao sódio, aumento de volemia,

1. Departamento de Clínica Médica, Universidade Estadual de Campinas, Campinas, São Paulo, Brasil.

2. Pronto Socorro Cardiológico de Pernambuco (PROCAPE), Recife, PE, Brasil.

3. MCor, Memorial São José Hospital - Rede D’ Or São Luiz, Recife, PE, Brasil.

Correspondência: Wilson Nadruz Junior, MD, Ph.D. Departamento de Clínica Médica Faculdade de Ciências Médicas, Universidade Estadual de Campinas Cidade Universitária “Zeferino Vaz", 13081-970 Campinas, SP. Brasil. wilnj@fcm.unicamp.br

http://dx.doi.org/10.47870/1519-7522/2020270130-3 
uso de substâncias exógenas levando a aumento da PA e causas secundárias (hiperaldosteronismo primário, síndrome da apneia e hipopneia obstrutiva do sono (SAHOS), estenose de artéria renal e doença renal crônica). . $^{1,3}$

Os pacientes que apresentam HAR mais frequentemente são afrodescendentes, idosos, sedentários, agregam diagnósticos concomitantes de diabetes mellitus, síndrome metabólica, nefropatia crônica e apresentam hipertrofia ventricular esquerda e albuminúria. ${ }^{1,3}$ A MAPA frequentemente mostra o efeito do jaleco branco (30\%) e ausência do descenso noturno. ${ }^{6}$

Fisiopatologicamente relaciona-se à HAR a hiperativação simpática e do sistema renina angiotensina aldosterona, proliferação da musculatura lisa vascular, retenção de sódio e maior ativação de fatores pró-inflamatórios. ${ }^{1,7}$

A HAR está relacionada à maior mortalidade e morbidade. Em um estudo retrospectivo com mais de 200.000 pacientes com hipertensão arterial sistêmica (HAS), aqueles com HAR tinham $47 \%$ mais chance de desfecho combinado de morte, infarto do miocárdio, insuficiência cardíaca, acidente vascular cerebral ou doença renal crônica ao longo de um seguimento de 3,8 anos. $^{8}$ Outro estudo comparou mais de 400.000 com e sem HAR, e mostrou aumento do risco em $32 \%$ para doença renal crônica, 24\% para infarto do miocárdio, 46\% para insuficiência cardíaca e $6 \%$ para morte associados à HAR. ${ }^{9}$

\section{BASE DO TRATAMENTO - OS TRÊS PRIMEIROS FÁRMACOS}

O tratamento inicial da HAR segue a mesma linha do tratamento inicial da HAS no momento do diagnóstico. As classes preconizadas para uso inicial são os diuréticos tiazídicos ou tipo-tiazídicos (TIAZ), os bloqueadores de canal de cálcio (BCC) e o bloqueio do sistema renina angiotensina aldosterona através dos inibidores da enzima conversora de angiotensina (IECA) ou dos bloqueadores dos receptores de angiotensina II (BRA).

A escolha de uma dessas três classes para o tratamento inicial foi comprovadamente eficaz no estudo ALLHAT, que randomizou mais de 41.000 pacientes hipertensos com pelo menos um fator de risco adicional de doença cardiovascular para quatro braços: clortalidona (doses de 12,5 a 25mg), lisinopril (doses de 10, 20 e $40 \mathrm{mg}$ ), anlodipino (doses de $2,5,5$ ou 10mg) ou doxazosina. O estudo no grupo com doxazosina foi interrompido precocemente devido a um aumento da incidência de insuficiência cardíaca. Os três braços restantes apresentaram resultados equivalentes na redução da PA e do desfecho primário de morte por doença cardiovascular ou infarto agudo do miocárdio não fatal. O grupo que recebeu clortalidona apresentou uma redução de $12,3 \mathrm{mmHg}$ na PA sistólica (PAS) e de $8,6 \mathrm{mmHg}$ na PA diastólica (PAD). O grupo que recebeu anlodipino apresentou uma redução de $12,5 \mathrm{mmHg}$ na PAS e de $9,3 \mathrm{mmHg}$ na PAD. Por fim, o grupo que recebeu lisinopril apresentou uma redução de $10,5 \mathrm{mmHg}$ na PAS e de $8,7 \mathrm{mmHg}$ na PAD. O desfecho primário combinado de morte cardiovascular e infarto agudo do miocárdio não fatal foi semelhante entre os grupos, sem diferenças estatisticamente significativas na comparação entre anlodipino e clortalidona [Risco Relativo (RR) 0,98; Intervalo de Confiança (IC) 95\% 0,90-1,07; p = 0,65] e entre lisinopril e clortalidona (RR 0,99; IC 95\% 0,91-1,08; $p=0.8) .{ }^{10}$
A combinação de drogas entre essas três classes é indicada em pacientes com HAS estágio 2 ou em caso de ausência de controle após o tratamento inicial em monoterapia. ${ }^{4} \mathrm{O}$ uso de duas drogas em doses menores também diminui os efeitos colaterais. ${ }^{11}$ Diversas combinações foram testadas e se mostraram igualmente eficazes. O uso de um BCC diidropiridinico de longa ação e um IECA, como no estudo ACCOMPLISH (anlodipino e benazepril) é sugerido como estratégia inicial, particularmente em pacientes com alto risco cardiovascular. ${ }^{12}$

A partir do uso de dois medicamentos iniciais, e em vigência de descontrole pressórico, a adição do terceiro leva a melhores controles de PA em 12-24 semanas. ${ }^{13}$ Vários estudos comprovaram a eficácia da combinação de um TIAZ, um BCC e um IECA/BRA. ${ }^{13-16}$ Entre as possibilidades de uso de TIAZ, a clortalidona e a indapamida apresentam superioridade em relação à hidroclorotiazida, uma vez que atingem melhor controle pressórico, possivelmente devido à sua meia-vida mais longa. ${ }^{17-19}$

\section{QUARTO FÁRMACO - ESPIRONOLACTONA}

A espironolactona é um antagonista dos receptores mineralocorticoides, com efeito diurético e anti-hipertensivo. Seu mecanismo de ação ocorre pela competição com aldosterona pelos sítios de receptores nos túbulos distais, aumentando a excreção de sódio e água e a retenção de potássio e hidrogênio. Seu pico de ação ocorre em 2,6 a 4,3 horas, com meia vida de 13,8 horas e excreção predominantemente renal. A dose usualmente utilizada para efeito anti-hipertensivo é de 12,5 a 50mg.

O uso de espironolactona como quarto fármaco no tratamento da HAR é recomendado pela 7̣a Diretriz Brasileira de Hipertensão Arterial, ${ }^{4}$ pelas Diretrizes atuais de HAS das Sociedades Europeias de Hipertensão e Cardiologia ${ }^{20}$ e da American Heart Association/American College of Cardiology. ${ }^{21}$

A eficácia da espironolactona em reduzir os níveis pressóricos quando utilizada como quarto fármaco (sendo os três primeiros um BCC, um BRA/IECA e um diurético) foi comprovada em diversos estudos, seja contra o placebo ${ }^{22}$ ou contra outros fármacos.

Entre eles, o estudo PATHWAY-2, um estudo randomizado, duplo cego e com crossover, avaliou 285 pacientes com diagnóstico de HAR randomizados para receber espironolactona (25-50mg), bisoprolol (5-10mg), doxazosina (4-8mg) e placebo durante 12 semanas. Conforme o desenho do estudo, os pacientes eram incluídos num esquema de rodizio das medicações, de forma que todos receberam cada uma das drogas por 12 semanas. Ao término do estudo, o desfecho primário de medidas de PA domiciliar foi favorável, com significância estatística, para a espironolactona, com média de diferença de redução de $8,7 \mathrm{mmHg}$ em relação ao placebo, 4,48mmHg em relação ao bisoprolol, 4,03mmHg em relação à doxazosina e $4,26 \mathrm{mmHg}$ em relação à média de redução com estes dois últimos fármacos. Os resultados foram consistentes ao longo dos grupos com diferentes níveis de renina plasmática, mas com maior margem de superioridade no grupo com maiores níveis de renina. Em 6 dos 285 pacientes que receberam espironolactona, o nível sérico de potássio ultrapassou $6,0 \mathrm{mmmol} / \mathrm{L}$ pelo menos uma vez. A taxa de efeitos colaterais (ginecomastia, insuficiência renal, hipercalemia) levando a suspensão do tratamento não foi 
maior no grupo da espironolactona em comparação às outras drogas e ao placebo. ${ }^{23}$

Uma meta análise publicada em 2016 incluiu 5 estudos randomizados e não randomizados (total de 502 pacientes) que comparavam o uso de espironolactona a outros fármacos (doxasozina, bisoprolol, furosemida e outra forma de bloqueio do sistema renina angiotensina aldosterona) como quarta droga no tratamento da HAR. O desfecho primário avaliado foi mudança na PAS medida no consultório ou em monitorização ambulatorial. O desfecho secundário foi mudança em níveis séricos de potássio e ocorrência de hipercalemia. O resultado encontrado foi que a espironolactona apresenta maior redução nos níveis de PAS quando comparado a outros fármacos, com média de 7,4 mmHg (IC 95\% 3,2-11,6). Quando a análise foi realizada apenas nos 3 estudos não randomizados, a média da redução foi ainda maior, de 11,9 mmHg (IC 95\% 9,3-14,4). A ocorrência de hipercalemia foi maior no grupo tratado com espironolactona (12 casos em 424 pacientes versus nenhum caso em 471 pacientes tratados com outras medicações). ${ }^{24}$

Esse efeito pode refletir, ao menos parcialmente, o fato de que pacientes com HAR apresentem níveis plasmáticos elevados de aldosterona em comparação a pacientes com PA normal ou hipertensos em uso de uma ou duas medicações. ${ }^{25}$ No entanto, a espironolactona é eficaz em reduzir a PA também em indivíduos com HAR que apresentem níveis plasmáticos e urinários normais de aldosterona. ${ }^{26}$

Os principais efeitos colaterais da espironolactona são hipercalemia, insuficiência renal aguda e ginecomastia. Em uma análise de um grupo do estudo Anglo-Scandinavian Cardiac Outcomes Trial (ASCOT), 1411 pacientes com descontrole pressórico a despeito do uso de três medicações anti-hipertensivas foram avaliados após a adição de espironolactona $25 \mathrm{mg} /$ dia. Houve redução média de 22/10 $\mathrm{mmHg}$ na PA após a adição do fármaco, com hipercalemia (potássio > $5,5 \mathrm{mEq} / \mathrm{L}$ ) ocorrendo em $4 \%$ dos pacientes e levando a $1 \%$ de taxa de suspensão da medicação. Ginecomastia foi registrada em $6 \%$ do total dos participantes, levando à descontinuidade do tratamento em $3 \%$ (todos homens). ${ }^{27}$

\section{QUARTO FÁRMACO - CLONIDINA}

A clonidina é um fármaco agonista dos receptores adrenérgicos alfa-2, que age através da redução do estímulo simpático do sistema nervoso central, levando à diminuição de resistência vascular periférica, resistência vascular renal, frequência cardíaca e pressão arterial. Como efeito anti-hipertensivo, tem pico de ação de 0,5 a 1,0 hora, meia vida de eliminação de
12-16 horas, metabolização hepática e excreção renal e dose usual de utilização de 0,2 a 0,6 mg/dia.

A clonidina foi introduzida em 1966 como uma alternativa de tratamento para a HAS. ${ }^{28}$ Sua eficácia no tratamento da HAS foi demonstrada em um estudo publicado no American Journal of Cardiology em 1971, no qual, comparado com o placebo, em doses de 0,3 a 0,6mg ao dia, levou à diminuição de cerca de $15 \mathrm{mmHg}$ na PAS e $10 \mathrm{mmHg}$ na PAD. ${ }^{29}$

Outro estudo, publicado em 2017, comparou o uso de clonidina com o uso de captopril para a situação específica de HAS pós-parto, sendo 90 pacientes randomizadas para cada grupo. Houve menos episódios de PA elevada durante a internação $(2,1$ versus $3,5 \%)$, maior porcentagem de redução de PA (14,0\% versus 10,8\%) e menos pacientes que necessitaram do uso de nitroprussiato de sódio (2,3\% versus $13,3 \%)$ no grupo que usou clonidina em relação ao grupo que utilizou captopril, mas essa diferença não foi estatisticamente significativa. ${ }^{30}$

Usualmente, no entanto, é uma droga reservada para tratamento de HAS refratária, ou associada ao tratamento da HAS quando seus efeitos colaterais (sonolência, sedação, boca seca) são favoráveis no auxílio do tratamento de outra patologia que o paciente apresente, como no caso de síndrome das pernas inquietas, retirada de opioides, flushes de menopausa e diarreia associada à neuropatia diabética. ${ }^{31-34}$

No entanto, até 2018, não haviam informações suficientes para sua comparação à espironolactona como quarto fármaco no tratamento da HAR.

No estudo Resistant Hypertension Optimal Treatment (ReHOT), publicado em 2018, 187 pacientes com HAR foram randomizados para a adição de espironolactona $12,5-50 \mathrm{mg} / \mathrm{dia}$ ou clonidina $0,1-0,3 \mathrm{mg}$ 12/12h. O desfecho primário foi o controle na PA de consultório $(<140 / 90 \mathrm{mmHg}$ ) ou na MAPA $(<130 / 80 \mathrm{mmHg})$. O desfecho primário foi atingido em 20,5\% dos pacientes com espironolactona e $20,8 \%$ dos pacientes com clonidina, sendo similar estatisticamente. No entanto, o grupo que recebeu espironolactona atingiu maior queda dos níveis de PAS e PAD em 24h em relação ao grupo que recebeu clonidina - redução em relação aos níveis basais de $11,8 \mathrm{mmHg}$ (IC 95\% 8,6 - 15,0) com espironolactona versus $7,3 \mathrm{mmHg}$ (IC 95\% 4,1 - 10,6) com clonidina para PAS e $6,3 \mathrm{mmHg}$ (IC 95\% 4,5 - 8,2) com espironolactona versus $3,9 \mathrm{mmHg}$ (IC 95\% 2,0 - 5,8) com clonidina para PAD (Tabela 1). A incidência de efeitos colaterais foi semelhante nos dois grupos, com a diferença de maior nível de creatinina plasmática no grupo espironolactona $(1,12$ versus $0,98 \mathrm{mg} / \mathrm{dL}$ ) e maior relato de sonolência no grupo de clonidina. A aderência não foi diferente entre os grupos. ${ }^{35}$

Tabela 1. Características farmacológicas da espironolactona e da clonidina e impacto destes fármacos sobre a medida da pressão arterial de 24 horas em pacientes com HAR com base no estudo ReHOT.

\begin{tabular}{|c|c|c|c|c|c|c|c|c|}
\hline Fármaco & $\begin{array}{l}\text { Mecanismo de } \\
\text { ação }\end{array}$ & $\begin{array}{l}\text { Dose } \\
\text { usual } \\
\text { (mg) }\end{array}$ & $\begin{array}{l}\text { Pico de } \\
\text { ação } \\
\text { (horas) }\end{array}$ & $\begin{array}{l}\text { Meia } \\
\text { vida } \\
\text { (horas) }\end{array}$ & Metabolização & Excreção & $\begin{array}{l}\text { Diminuição da PA } \\
\text { sistólica (ReHOT) }\end{array}$ & $\begin{array}{l}\text { Diminuição da } \\
\text { PA diastólica } \\
\text { (ReHOT) }\end{array}$ \\
\hline Espironolactona & $\begin{array}{l}\text { Antagonista } \\
\text { do receptor } \\
\text { mineralocorticoide }\end{array}$ & $12,5-50$ & $2,6-4,3$ & 13,8 & Hepática & Renal & $\begin{array}{l}11,8 \mathrm{mmHg}(\mathrm{IC} 95 \% \\
8,6-15,0)\end{array}$ & $\begin{array}{l}\text { 6,3 } \mathrm{mmHg} \\
\text { (IC 95\% 4,5-8,2) }\end{array}$ \\
\hline Clonidina & $\begin{array}{l}\text { Agonista } \\
\text { do receptor } \\
\text { adrenérgico alfa-2 }\end{array}$ & $0.2-0,6$ & $0,5-1,0$ & $12-16$ & Hepática & Renal & $\begin{array}{l}\text { 7,3 mmHg } \\
\text { (IC 95\% 4,1 - 10,6) }\end{array}$ & $\begin{array}{l}3,9 \mathrm{mmHg} \\
\text { (IC 95\% 2,0-5,8) }\end{array}$ \\
\hline
\end{tabular}




\section{CONCLUSÃO}

O uso da espironolactona como quarto fármaco no tratamento da HAR é recomendado pelas principais diretrizes de tratamento de HAS, e tem a sua eficácia comprovada em estudos randomizados e meta-análises. A alternativa do uso de clonidina como quarta droga, conforme testado no estudo
ReHOT, apresenta reduções inferiores nos níveis de PA de 24h, mas pode ser uma escolha razoável em pacientes que não toleram antagonistas de receptores de mineralocorticoides, como os que apresentam hipercalemia ou doença renal crônica pré-dialítica antes do início do tratamento.

\section{REFERÊNCIAS}

1. Calhoun DA, Jones D, Textor S, et al. Resistant Hypertension: Diagnosis, Evaluation, and Treatment A Scientific Statement From the American Heart Association Professional Education Committee of the Council for High Blood Pressure Research. Circulation. 2008;117(25):e510-26.

2. Judd E, Calhoun DA. Apparent and true resistant hypertension: definition, prevalence and outcomes. J Hum Hypertens. 2014;28(8):463-8.

3. Alessi A, Brandao AA, Coca A et al. First Brazilian Position on Resistant Hypertension; Arq. Bras Cardiol. 2012;99(1):576-85.

4. Malachias MVB, Gomes MAM, Nobre F el at. VII Diretriz Brasileira de Hipertensão Arterial. Arq Bras Cardiol 2016. 107( 3 Suppl 3 ): 7-13.

5. Pedrosa RP, Drager LF, Gonzaga CC, et al. Obstructive Sleep Apnea The Most Common Secondary Cause of Hypertension Associated With Resistant Hypertension. Hypertension. 2011;58;811-7.

6. De La Sierra A, Segura J, Banegas JR, et al. Clinical features of 8295 patients with resistant hypertension classified on the basis of ambulatory blood pressure monitoring. Hypertension. 2011;57(5):898-902.

7. de Faria AP, Modolo R, Fontana V. Moreno H. Adipokines: Novel Players in Resistant Hypertension. J Clin Hypertens. 2014;16(10):754-9.

8. Daugherty SL, David Powers J, Magid DJ, et al. Incidence and Prognosis of Resistant Hypertension in Hypertensive Patients. Circulation. 2012;125(13):1635-42.

9. Sim JJ, Bhandari SK, Shi J, et al. Comparative risk of renal, cardiovascular, and mortality outcomes in controlled, uncontrolled resistant, and non-resistant hypertension. Kidney Int. 2015;88(3): 622-32.

10. ALLHAT Officers and Coordinators for the ALLHAT Collaborative Research Group. The Antihypertensive and Lipid-Lowering Treatment to Prevent Heart Attack Trial.. Major outcomes in high-risk hypertensive patients randomized to angiotensin-converting enzyme inhibitor or calcium channel blocker vs diuretic: The antihypertensive and lipid-lowering treatment to prevent heart attack trial (ALLHAT). JAMA. 2002;288(23):2981-2997.

11. Bangalore S, Kamalakkannan G, Parkar S, Messerli FH. Fixed-Dose Combinations Improve Medication Compliance: A Meta-Analysis. Am J Med. 2007;120(8):713-9.

12. Jamerson $\mathrm{K}$, Weber MA, Bakris GL, et al. Benazepril plus amlodipine or hydrochlorothiazide for hypertension in high-risk patients. N Engl J Med. 2008;359(23):2417-28.

13. Chrysant SG, Littlejohn T 3rd, Izzo JLJr, et al. Triple-Combination therapy with olmesartan, amlodipine, and hydrochlorothiazide in black and non-black study participants with hypertension: the TRINITY randomized, double-blind, 12-week, parallel-group study. Am J Cardiovasc Drugs. 2012 ;12(4):233-43.

14. Calhoun DA, Lacourcie Y, Chiang YT, Glazer RD. Triple Antihypertensive Therapy With Amlodipine, Valsartan, and Hydrochlorothiazide. Hypertension. 2009:54(1)32-9.

15. Düsing R, Waeber B, Destro M, Santos Maia C, Brunel P. Triple-combination therapy in the treatment of hypertension: A review of the evidence. J Hum Hypertens. 2017;31(8):501-10.

16. Webster R, Salam A, Silva HA De, et al. Fixed Low-Dose Triple Combination Antihypertensive Medication vs Usual Care for Blood Pressure Control in Patients With Mild to Moderate Hypertension in Sri Lanka A Randomized Clinical Trial. JAMA. 2018;320(6):566-79.

17. Khosla N, Chua DY, Elliott WJ, Bakris GL. Are Chlorthalidone and Hydrochlorothiazide Equivalent Blood-Pressure-Lowering Medications? J Clin Hypertens. 2005;7(6):354-6.

18. Sica DA. Chlorthalidone: Has it always been the best thiazide-type diuretic? Hypertension. 2006;47(3):321-2.

19. Ernst ME, Carter BL, Zheng S, Grimm RH. Meta-Analysis of Dose-Response Characteristics of Hydrochlorothiazide and Chlorthalidone: Effects on Systolic Blood Pressure and Potassium. Am J Hypertens. 2010;23:440-6.

20. Williams B, Mancia G, Spiering W, et al. 2018 Practice Guidelines for the management of arterial hypertension of the European Society of Hypertension and the European Society of Cardiology: ESH/ESC Task Force for the Management of Arterial Hypertension. J Hypertens. 2018;36:2284-309.

21. Whelton PK, Carey RM, Aronow WS, et al. Clinical Practice Guideline 2017 ACC / AHA / AAPA / ABC / ACPM/AGS/APhA/ASH/ASPC/NMA/PCNA Guideline for the Prevention, Detection, Evaluation, and Management of High Blood Pressure in Adults A Report of the American College of Cardiology / American Heart Association Task Force on Clinical Practice Guidelines. J Am ColL Cardiol. 2018;71(19):e127-248.

22. Vaclavik J, Sedlak R, Jarjovsky J, Kociánová E, Táborský M. Effect of spironolactone in resistant arterial hypertension: a randomized, doubleblind, placebo-controlled trial (ASPIRANT-EXT). Medicine (Baltimore) 2014; 93(27):e162.

23. Williams B, Macdonald TM, Morant S, et al. Spironolactone versus placebo, bisoprolol, and doxazosin to determine the optimal treatment for drug-resistant hypertension (PATHWAY-2): A randomised, double-blind, crossover trial. Lancet. 2015;386(10008):2059-68.

24. SinnottSJ, Tomlinson LA, RootAA, et al. Comparative effectiveness of fourthline anti-hypertensive agents in resistant hypertension: A systematic review and meta-analysis. Eur J Prev Cardiol. 2017;24(3):228-38.

25. Gaddam KK, Nishizaka MK, Pratt-Ubunama MN, et al. Characterization of resistant hypertension: Association between resistant hypertension, aldosterone, and persistent intravascular volume expansion. Arch Intern Med. 2008;168(11):1159-64.

26. Nishizaka MK, Zaman MA, Calhoun DA. Efficacy of Low-Dose Spironolactone in Subjects With Resistant Hypertension. Am J Hypertens. 2003; 16:925-30.

27. Chapman N, Dobson J, Wilson S, et al. Effect of spironolactone on blood pressure in subjects with resistant hypertension. Hypertension. 2007;49(4):839-45.

28. Stähle H. A historical perspective: Development of clonidine. Best Pract Res Clin Anaesthesiol. 2000;14(2):237-246.

29. Hoobler SW, Sagastume E. Clonidine hydrochloride in the treatment of hypertension. Am J Cardiol. 1971;28(1):67-73.

30. Noronha Neto C C, Maia SSB, Katz L, Coutinho IC, Souza AR, Amorim MM. Clonidine versus Captopril for Severe Postpartum Hypertension: A Randomized Controlled Trial. PLoS One. 2017;12(1):e0168124.

31. Wagner ML, Walters S, Coleman RG, Hening A, Grasing K, Chokroverty S. Randomized, double-blind, placebo-controlled study of clonidine in restless legs syndrome. 1996;19(1):52-8.

32. Bond WS. Psychiatric indications for clonidine: the neuropharmacologic and clinical basis. J Clin Psychopharmacol. 1986;6(2):81-7.

33. Pandya KJ, Raubertas RF, Flynn PJ, et al. Oral clonidine in postmenopausal patients with breast cancer experiencing tamoxifen-induced hot flashes: A University of Rochester Cancer Center Community Clinical Oncology Program study. Ann Intern Med. 2000;132(10):788-93.

34. Fedorak RN, Field M, Chang EB. Treatment of Diabetic Diarrhea with Clonidine. Ann Inter Med. 1985;102(2):197-9.

35. Krieger EM, Drager LF, Giorgi DMA, et al. Resistant Hypertension Optimal Treatment Trial ( ReHOT) Spironolactone Versus Clonidine as a Fourth-Drug Therapy for Resistant Hypertension. Hypertension. 2018; 71(4) :681-90. 